PROCEEDINGS OF THE

AMERICAN MATHEMATICAL SOCIETY

Volume 138, Number 3, March 2010, Pages 787-797

S 0002-9939(09)10189-2

Article electronically published on October 23, 2009

\title{
THE CARDINALITY OF SOME SYMMETRIC DIFFERENCES
}

\author{
PO-YI HUANG, WEN-FONG KE, AND GÜNTER F. PILZ
}

(Communicated by Jim Haglund)

\begin{abstract}
In this paper, we prove that for positive integers $k$ and $n$, the cardinality of the symmetric differences of $\{1,2, \ldots, k\},\{2,4, \ldots, 2 k\},\{3,6, \ldots, 3 k\}$, $\ldots,\{n, 2 n, \ldots, k n\}$ is at least $k$ or $n$, whichever is larger. This solved a problem raised by Pilz in which binary composition codes were studied.
\end{abstract}

\section{INTRODUCTION}

The symmetric difference of two sets $A$ and $B$, denoted by $A \Delta B$, is $(A \backslash B) \cup(B \backslash$ $A)$. For any positive integers $k$ and $n$, the cardinality of the symmetric difference

$$
\{1,2, \ldots, k\} \Delta\{2,4, \ldots, 2 k\} \Delta \cdots \Delta\{n, 2 n, \ldots, k n\}
$$

is of interest in several different situations. Here we mention three of them:

- "To love or not to love". Let us take $k=3$. Suppose three people with numbers 1,2 , and 3 on their back enter an empty room. Then three more people with numbers 2,4 and 6 go into this room. Now two people have the same number, namely 2; they fall in love and leave the room. So only numbers 1, 3, 4 and 6 remain. Next, people with numbers 3,6 and 9 come in. Numbers 3 and 6 find partners, and only the three people with numbers 1, 4 and 9 remain, and so on. The conjecture is: "There will always be at least 3 people in the room." This is easy to show, but it seems considerably harder for a general $k$ greater than 3 .

- "Summands in binary polynomials". Over $\mathbb{Z}_{2}$, consider the sum of polynomials

$$
\begin{aligned}
\left(1+x+\cdots+x^{k}\right) \circ x+(1+x+ & \left.\cdots+x^{k}\right) \circ x^{2} \\
& +\cdots+\left(1+x+\cdots+x^{k}\right) \circ x^{n} \\
=\left(1+x+\cdots+x^{k}\right)+\left(1+x^{2}+\cdots\right. & \left.+x^{2 k}\right) \\
& +\cdots+\left(x^{n}+x^{2 n}+\cdots+x^{k n}\right) .
\end{aligned}
$$

Received by the editors June 1, 2009.

2010 Mathematics Subject Classification. Primary 05A05; Secondary 11N05, 94B05.

The first author was supported by the National Science Council, Taiwan, grant \#96-2115-M006-003-MY3.

The second author was partially supported by the National Science Council, Taiwan, grant \#97-2923-M-006-001-MY2.

The third author was supported by grant P19463 of the Austrian National Science Fund (FWF).

(C)2009 American Mathematical Society Reverts to public domain 28 years from publication 
Will there always be at least $k$ summands present? Equivalently, has the symmetric difference

$$
\{1,2, \ldots, k\} \Delta\{2,4, \ldots, 2 k\} \Delta \cdots \Delta\{n, 2 n, \ldots, k n\}
$$

always at least $k$ elements?

- "Codes by composition". Encode a binary message $\left(a_{1}, a_{2}, \ldots, a_{n}\right)$ of length $n$ as a polynomial by composition as

$a_{1}\left(1+x+\cdots+x^{k}\right) \circ x+a_{2}\left(1+x+\cdots+x^{k}\right) \circ x^{2}+\cdots+a_{n}\left(1+x+\cdots+x^{k}\right) \circ x^{n}$.

There is a good reason to do this kind of coding; see 3 . A positive answer in item 2 would give a positive indication that the minimal weight of these codewords is $k$.

With some extensive experimental data, the following conjecture, with a convenient name, was raised in [3].

1-2-3 Conjecture. The cardinality of the symmetric difference

$$
\{1,2, \ldots, k\} \Delta\{2,4, \ldots, 2 k\} \Delta \cdots \Delta\{n, 2 n, \ldots, k n\}
$$

is always at least $k$.

It was shown in 3 that the conjecture holds true for $k \leq 6$. In private communications, E. Fried (Budapest) proved it for $k=7$ and $k=8$, and P. Fuchs (Linz) for $k \geq 10^{12}$.

In this paper, we prove a slightly general version of the conjecture. Some notation can be useful for our discussion.

For $k, s \in \mathbb{N}$, let $I_{k}=\{1,2, \ldots, k\}$ and $s I_{k}=\{s, 2 s, \ldots, k s\}$. For $1 \leq u<v$, put $D_{k \times[u, v]}=u I_{k} \Delta(u+1) I_{k} \Delta \cdots \Delta v I_{k}$. When $u=1$, we use $D_{k \times v}$ instead of $D_{k \times[1, v]}$ and denote by $d_{k}(n)$ the cardinality of $D_{k \times n}$. It is obvious that if $1<s<n$, then $D_{k \times n}=D_{k \times s} \Delta D_{k \times[s+1, n]}$. Also,

$$
D_{k \times n}=D_{n \times k} \text { for all } k \text { and } n .
$$

Now, we modify the conjecture (but still keep the same name) and shorten it using the prepared notation.

1-2-3 Conjecture. For all $k, n \in \mathbb{N}, d_{k}(n) \geq \max \{n, k\}$.

It is easy to see that $D_{k \times k}=\left\{1^{2}, 2^{2}, \ldots, k^{2}\right\}$, which has $k$ elements. The fact (1:1) tells us that it suffices to show

Restricted 1-2-3 Conjecture. For all $n>k$, it follows that $d_{k}(n) \geq n$.

In this paper, we will show that this conjecture has a positive answer.

\section{THE CASE WHEN $k<n \leq 2 k$}

Let $k$ and $w$ be fixed such that $1 \leq w \leq k$, and let $n=k+w$. First, we make two general observations:

(1) For any positive integer $a$, the set $a I_{k}$ contains at most $\lfloor\sqrt{k}\rfloor$ many squares. To see this, we notice that the greatest common divisor $u$, say, of the squares in $a I_{k}$ is itself a square and is a multiple of $a$. Hence $u \in a I_{k}$. Therefore, the squares contained in $a I_{k}$ are contained in $\left\{u, 4 u, 9 u, \ldots,\lfloor\sqrt{k}\rfloor^{2} u\right\}$, and so there are at most $\lfloor\sqrt{k}\rfloor$ of them. 
(2) Let $s$ and $t$ be distinct integers with $k<s \leq w$ and $k<t \leq w$. Then $s t \notin s I_{k}$ and $s t \notin t I_{k}$ since $s t>s k$ and $s t>t k$. Suppose that $\ell$ is the greatest common divisor of $s$ and $t$. Then $\ell \leq|t-s| \leq w-1$, and

$$
s I_{k} \cap t I_{k} \subseteq\{s t / \ell, 2 s t / \ell, \ldots,(\ell-1) s t / \ell\} .
$$

Therefore, $\left|s I_{k} \cap t I_{k}\right| \leq \ell-1$, and the number of cancellations taking place in $s I_{k}$ and $t I_{k}$ is at most $2(\ell-1)$.

Since $D_{k \times k}=\left\{1^{2}, 2^{2}, \ldots, k^{2}\right\}$, and the number of squares in $(k+1) I_{k}$ is less than or equal to $\sqrt{k}$, we have $d_{k}(k+1) \geq 2 k-2 \sqrt{k}$. Also, since $k+1$ and $k+2$ are coprime to each other, $(k+1) I_{k}$ and $(k+2) I_{k}$ do not have anything in common. Thus $d_{k}(k+2) \geq 3 k-4 \sqrt{k}$. Now, $k+2$ and $k+3$ are also coprime, and $(k+1) I_{k}$ and $(k+3) I_{k}$ have at most one element in common. We have $d_{k}(k+3) \geq 4 k-6 \sqrt{k}-2$. Finally, counting in the possible cancellations among $(k+1) I_{k},(k+2) I_{k},(k+3) I_{k}$, and $(k+4) I_{k}$, we have $d_{k}(k+4) \geq 5 k-8 \sqrt{k}-6$. Thus, for $w=1,2,3$, 4 , we want

$$
\begin{aligned}
2 k-2 \sqrt{k} & \geq k+1, \\
3 k-4 \sqrt{k} & \geq k+2, \\
4 k-6 \sqrt{k}-2 & \geq k+3, \\
5 k-8 \sqrt{k}-6 & \geq k+4 .
\end{aligned}
$$

As long as $k \geq 9$, the above inequalities hold.

In the following, we assume that $k \geq 9$ and $w \geq 5$. For $2 \leq \ell \leq w-1$, put

$$
C_{\ell}=\{a \mid \ell \text { divides } a, \text { and } k+1 \leq a \leq k+w\} .
$$

Then $\left|C_{\ell}\right| \leq\left\lceil\frac{w}{\ell}\right\rceil$, and so the number of cancellations among the $a I_{k}$ 's, $a \in C_{\ell}$, can be no more than

$$
\left(\begin{array}{c}
\left\lceil\frac{w}{\ell}\right\rceil \\
2
\end{array}\right) \cdot 2(\ell-1)=\left\lceil\frac{w}{\ell}\right\rceil\left(\left\lceil\frac{w}{\ell}\right\rceil-1\right)(\ell-1) \leq\left(\frac{w}{\ell}+1\right) \frac{w}{\ell}(\ell-1)<\left(\frac{w}{\ell}+1\right) w .
$$

Therefore, the total number of cancellations occurring in $(k+1) I_{k}, \ldots,(k+w) I_{k}$ is at most

$$
\begin{aligned}
\sum_{\ell=2}^{w-1}\left(\begin{array}{c}
\left\lceil\frac{w}{\ell}\right\rceil \\
2
\end{array}\right) \cdot 2(\ell-1)<\sum_{\ell=2}^{w-1}\left(\frac{w}{\ell}+1\right) w & <\sum_{\ell=2}^{w-1}\left(\frac{w}{\ell}+\frac{w}{\ell}\right) w \\
= & \sum_{\ell=2}^{w-1} \frac{2 w^{2}}{\ell}=2 w^{2} \sum_{\ell=2}^{w-1} \frac{1}{\ell}<2 w^{2} \cdot \ln (w-1) .
\end{aligned}
$$

After cancellations there are at least $k w-2 w^{2} \ln (w-1)$ many elements left in $D_{k \times[k+1, k+w]}$.

Now, $D_{k \times(k+w)}$ has at least $2 k$ elements as long as $k w-2 w^{2} \cdot \ln (w-1) \geq 3 k$, or equivalently,

$$
k \geq \frac{2 w^{2} \cdot \ln (w-1)}{w-3} .
$$

Note that the function $f(x)=\frac{2 x^{2} \cdot \ln (x-1)}{x-3}$ is increasing for $x \geq 5$. For each given $k$, let $w_{k}=\max \{w \geq 1 \mid w$ satisfies $(2: 1)\}$. Table 1 gives various $k$ and $w_{k}$. 
TABLE 1

\begin{tabular}{|c|c|c|c|c|c|c|c|}
\hline$k$ & $w_{k}$ & $k$ & $w_{k}$ & $\bar{k}$ & $w_{k}$ & $k$ & $w_{k}$ \\
\hline 35 & 5 & 100 & 15 & 1000 & 105 & 10000 & 753 \\
\hline 50 & $\overline{8}$ & 200 & 27 & 2000 & 189 & 20000 & 1381 \\
\hline 70 & $\overline{11}$ & 300 & 38 & 4000 & 341 & 40000 & 2548 \\
\hline 90 & $\overline{13}$ & 600 & 68 & 8000 & 620 & 80000 & 4725 \\
\hline
\end{tabular}

From Table 1 we know that, for example, if $k=70$, then each of the sets $D_{k \times(k+s)}, 5 \leq s \leq 11$, has at least $2 k=140$ elements in there. As another example, let $k=40000$. Then each of the sets $D_{k \times(k+s)}, 5 \leq s \leq 2548$, has at least 80000 elements in there.

We note that if a prime $p$ occurs in $\{k+1, \ldots, k+s\}, 1 \leq s \leq k$, then $D_{k \times(k+s)}$ has at least $k$ elements in it as the elements of $p I_{k}$ cannot be canceled. To see this, we assume that $p s=q t \in p I_{k} \cap q I_{k}$ for some integer $q>k$ with $q \neq p$, and $s, t \in I_{k}$. Then $p$ divides $q$ since $t \leq k<p$. From $p>k$, we infer that $q>2 k$.

Lemma 2.1. Suppose that $w_{k} \geq 5$ and that there are two distinct primes among $k+1, \ldots, k+w_{k}$. Then $D_{k \times(k+s)}$ has at least $2 k$ elements for all $s$ with $5 \leq s \leq k$.

Proof. If $5 \leq s \leq w_{k}$, then we have seen from $k \geq \frac{2 s^{2} \cdot \ln (s-1)}{s-3}$ that $D_{k \times(k+s)}$ has at least $2 k$ elements. On the other hand, if $w_{k}<s \leq k$, then the two primes between $k+1$ and $k+w_{k}$ give us what we want.

Therefore, we assume that $w_{k} \geq 5$, and we would like to have two distinct primes among $k+1, k+2, \ldots, k+w_{k}$. This brings us to the prime gaps consideration.

A prime gap is the difference between two successive prime numbers. The $n$-th prime gap is the difference between the $(n+1)$-th and the $n$-th prime number. One writes $g(p)$ for the the gap $q-p$, where $q$ is the next prime to $p$. A prime gap is said to be maximal if it is larger than all gaps between smaller primes. The notation for the $n$-th maximal prime gap is $g_{n}$. Table 2 shows $g_{n}$ for $1 \leq n \leq 15$. For example, for any prime $p$ less than 9551, the prime gap $g(p)$ is less than 36 . That is to say that for any prime $p$ with $p<9551$, there must be a prime in the set $\{p, p+1, \ldots, p+35\}$.

TABLE 2

\begin{tabular}{|c|c|c|c|c|c|c|c|c|}
\hline$n$ & $g_{n}$ & $p_{n}$ & $n$ & $g_{n}$ & $p_{n}$ & $n$ & $g_{n}$ & $p_{n}$ \\
\hline 1 & 1 & 2 & 6 & 14 & 113 & 11 & 36 & 9551 \\
\hline 2 & 2 & 3 & 7 & 18 & 523 & 12 & 44 & 15683 \\
\hline 3 & 4 & 7 & 8 & 20 & 887 & 13 & 52 & 19609 \\
\hline 4 & 6 & 23 & 9 & 22 & 1129 & 14 & 72 & 31397 \\
\hline 5 & 8 & 89 & 10 & 34 & 1327 & 15 & 86 & 155921 \\
\hline
\end{tabular}

If $p$ is a prime with $p>2 k$ and the maximal prime gap $g_{m}=g(q)<\frac{w_{k}}{2}$, where $q$ is the first prime to have $g(q)=g_{m}$, then there must exist at least two primes among $k+1, k+2, \ldots, k+w_{k}$. 
Take $k=300$; then $w_{k}=38$. Now, 601 is the prime just bigger then $2 k=600$, and the maximal prime gap for primes less than 887 is at most $g_{7}=18$. Thus, $g(601) \leq 18<\frac{w_{k}}{2}$. By Lemma 2.1. $D_{k \times(k+s)}$ contains at least $2 k=600$ elements for any $s$ with $5 \leq s \leq 300$.

For those $k$ which are less than 300, we can check easily using GAP [1] that $d_{k}(n) \geq n$ for $k<n \leq 2 k$. Actually, a small program in GAP running on a modern $\mathrm{PC}$ takes about 90 seconds to verify it.

For those $k$ that are greater than 300 , we will argue in the following that $d_{k}(n) \geq$ $n$ for $k<n \leq 2 k$ by using the monotone increasing property of $w_{k}$ and maximal prime gaps.

In [4, p. 368], one finds

$$
\text { for } n \geq 2, \quad p_{n} \geq n(\ln n+\ln \ln n-1.0072629)
$$

and

$$
\text { for } n \geq 7022, \quad p_{n} \leq n(\ln n+\ln \ln n-0.9385) .
$$

Therefore, for $n \geq 7022$, we have

$$
(n+1) \ln (n+1)-n \ln n=n \ln \left(\frac{1}{n}+1\right)+\ln (n+1) \leq n \cdot \frac{1}{n}+\ln (n+1)=1+\ln (n+1)
$$

and

$$
\begin{aligned}
(n+1) \ln \ln (n+1)-n \ln \ln n & =n \ln \left(\frac{\ln (n+1)}{\ln n}\right)+\ln \ln (n+1) \\
& \leq n \cdot\left(\frac{\ln (n+1)}{\ln n}-1\right)+\ln \ln (n+1) \\
& \leq n \cdot\left(\frac{\ln 7023}{\ln 7022}-1\right)+\ln \ln (n+1) \\
& <0.00002 n+\ln \ln (n+1) .
\end{aligned}
$$

The last two inequalities hold since the function $f(x)=\frac{\ln (x+1)}{\ln x}$ is decreasing and $\frac{\ln 7023}{\ln 7022}$ is less than 1.00002 . Hence

$$
\begin{aligned}
g\left(p_{n}\right) & =p_{n+1}-p_{n} \\
& \leq(n+1)(\ln (n+1)+\ln \ln (n+1)-0.9385)-n(\ln n+\ln \ln n-1.0072629) \\
& \leq(1+\ln (n+1))+(0.00002 n+\ln \ln (n+1))+0.0687629 n-0.9385 .
\end{aligned}
$$

As $\ln x+\ln \ln x$ is increasing and concave downward, we have

$$
\begin{aligned}
& \ln (n+1)+\ln \ln (n+1) \\
\leq & (\ln 7023+\ln \ln 7023)+\left.(\ln x+\ln \ln x)^{\prime}\right|_{7023} \cdot(n-7022) \\
< & \ln 7023+\ln \ln 7023+0.0001585 n-1.1 \\
< & 11+0.0001585 n,
\end{aligned}
$$

and so

$$
g\left(p_{n}\right)=p_{n+1}-p_{n}<12+0.069 n<0.071 n \text { for } n \geq 7022 .
$$

Now we consider $D_{k \times n}$ with $k>p_{7022}=70919$ and $k+5 \leq n=k+w \leq 2 k$. If $k \geq \frac{2 w^{2} \ln (w-1)}{w-3}$, then $d_{k}(n) \geq 2 k \geq n$ as we have seen in Lemma 2.1. Thus, suppose that $k<\frac{2 w^{2} \ln (w-1)}{w-3}$. Since $k>70919$, we certainly have $w>9$, and 
so $\ln \ln (3 w)=\ln (\ln 3+\ln w)>\ln (3 \ln 3)>0$. Also, from $w^{2}-9 w>0$, we get $2 w^{2}<3 w(w-3)$. Combining these with $\ln 3>1.098$ (hence $\ln 3-1.0072629>0$ ), we get

$$
\begin{aligned}
2 w^{2} \ln (w-1) & <3 w(w-3) \ln w \\
& <3 w(w-3)(\ln w+\ln 3-1.0072629+\ln \ln (3 w)) \\
& <3 w(w-3)(\ln (3 w)+\ln \ln (3 w)-1.0072629) \\
& \leq(w-3) p_{3 w}
\end{aligned}
$$

by (2:2). Therefore,

$$
p_{7022}<k<\frac{2 w^{2} \ln (w-1)}{w-3}<p_{3 w} .
$$

As $g\left(p_{3 w}\right)<0.213 w$ by (2:4), the prime gaps for the primes between $p_{7022}$ and $p_{3 w}$ are all smaller than $0.213 w$. This means that there is a prime between $k$ and $k+0.213 w$, and another one between $k+0.213 w$ and $k+2 \cdot 0.213 w$. In particular, there are at least two distinct primes in $[k, k+w]$, and so $D_{k}(n) \geq 2 k \geq n$ again by Lemma 2.1 .

Next, suppose that $k \leq p_{7022}$. From the prime gap table above, we see that for any prime $p$ with $k \leq p \leq p_{7022}=70919<155928$, the prime gap $g(p)$ is less than or equal to $g_{14}=72$. Also, for $2000<k \leq 70919$, we have $w_{k} \geq 189>144$. Thus, there are at least two primes between $k$ and $k+w_{k}$.

For any $k$ with $600<k \leq 2000, w_{k} \geq 68$. For any prime $p$ with $k \leq p \leq 2000<$ 9551 , the prime gap $g(p)$ is at most 34 . Thus, for any $k$ with $600<k \leq 2000$, there are at least two primes between $k$ and $k+w_{k}$.

For $k$ with $300<k \leq 600, w_{k} \geq 38$, and for any prime $p$ with $k \leq p \leq 600<887$, the prime gap $g(p)$ is at most 18 . Again, for $k$ with $300<k \leq 600$, there are at least two primes between $k$ and $k+w_{k}$.

Therefore, for all $k$ with $300<k \leq p_{7022}$, there are always two primes between $k$ and $k+w_{k}$. By Lemma 2.1, $d_{k}(n) \geq 2 k>n$ for any such $k$ and any $n$ with $k+5 \leq n \leq 2 k$.

We have now shown that $d_{k}(n)>n$ for all $k$ and $n$ with $k>300$ and $k<n \leq 2 k$. Hence we can announce that the Restricted 1-2-3 Conjecture is true for any $n, k \in \mathbb{N}$ with $k<n \leq 2 k$.

Induction kicks in from here. The starting ground is that $d_{1}(n)=n$ for all $n>1$. Let $k>1$, and assume that we have the Restricted 1-2-3 Conjecture verified up to $k-1$. That is, assume that $d_{s}(m) \geq m$ when $s \leq k-1<m$. We want to show that $d_{k}(n) \geq n$ for all $n>k$. From the above, we also know that this is true for $n$ up to $2 k$. So, we assume that $n>2 k$ and also that $d_{k}(m) \geq m$ when $2 k \leq m<n$. And we continue....

\section{A REDUCTION}

The first step is to make certain that we do not need to care too much for $n$ large enough. Namely, we will show that it is sufficient to restrict $n$ to be no larger than $\operatorname{LCM}\left(I_{k}\right)$, the least common multiple of $\{1,2, \ldots, k\}$. 
Let $T$ be a nonempty subset of $I_{k}$ with $|T|=\ell$. Then we have

$$
\begin{aligned}
\sum_{\varnothing \neq S \subset T}(-2)^{|S|-1} & =\sum_{i=1}^{\ell}\left(\begin{array}{l}
\ell \\
i
\end{array}\right)(-2)^{i-1} \\
& =(-2)^{-1} \cdot \sum_{i=1}^{\ell}\left(\begin{array}{l}
\ell \\
i
\end{array}\right)(-2)^{i} \\
& =(-2)^{-1} \cdot\left(((-2)+1)^{\ell}-1\right) \\
& =(-2)^{-1} \cdot\left((-1)^{\ell}-1\right) \\
& = \begin{cases}0, & \text { if } \ell \text { is even; } \\
1, & \text { if } \ell \text { is odd. }\end{cases}
\end{aligned}
$$

For convenience and by abusing notation, if $T=\varnothing$, we put $\sum_{\varnothing \neq S \subset T}(-2)^{|S|-1}=0$. Using this identity, we have

Theorem 3.1. Let $n \geq k$. Then

$$
d_{k}(n)=\sum_{\varnothing \neq S \subset I_{k}}\left\lfloor\frac{n \cdot \min S}{\operatorname{LCM}(S)}\right\rfloor \cdot(-2)^{|S|-1} .
$$

Proof. Denote by $2^{I_{k}}$ the power set of $I_{k}$. Define $\theta: \mathbb{N} \rightarrow 2^{I_{k}}$ by $\theta(m)=\left\{s \in I_{k} \mid\right.$ $\left.m \in s I_{n}\right\}$. For $\varnothing \neq S \subseteq I_{k}$, set

$$
\widetilde{S}=\theta^{-1}(S)=\{m \in \mathbb{N} \mid \theta(m)=S\}
$$

and

$$
\bar{S}=\{m \in \mathbb{N} \mid \theta(m) \supseteq S\} .
$$

Note that if $\varnothing \neq S \subseteq T$, then $m \in \bar{S}$ whenever $m \in \widetilde{T}$, and $\bar{S}$ is the disjoint union of $\widetilde{T}$ for subsets $T$ of $I_{k}$ containing $S$. Therefore, $|\bar{S}|=\sum_{S \subseteq T \subseteq I_{k}}|\widetilde{T}|$.

An integer $m \geq 1$ will appear in $D_{k \times n}$ if and only if $\theta(m)$ is a nonempty set with an odd number of elements in it. Therefore, we have

$$
\begin{aligned}
d_{k}(n) & =\sum_{m \geq 1}\left(\sum_{\varnothing \neq S \subseteq \theta(m)}(-2)^{|S|-1}\right) \\
& =\sum_{\varnothing \neq T \subseteq I_{k}}|\widetilde{T}| \cdot\left(\sum_{\varnothing \neq S \subseteq T}(-2)^{|S|-1}\right) \\
& =\sum_{\varnothing \neq T \subseteq I_{k}} \sum_{\varnothing \neq S \subseteq T}|\widetilde{T}| \cdot(-2)^{|S|-1} \\
& =\sum_{\varnothing \neq S \subseteq I_{k}}\left(\sum_{S \subseteq T \subseteq I_{k}}|\widetilde{T}|\right) \cdot(-2)^{|S|-1} \\
& =\sum_{\varnothing \neq S \subseteq I_{k}}|\bar{S}| \cdot(-2)^{|S|-1} .
\end{aligned}
$$

To finish the proof, we notice that for any nonempty subset $S$ of $I_{k}$,

$$
\begin{aligned}
\bar{S} & =\left\{m \geq 1 \mid m \in s I_{n} \text { for all } s \in S\right\} \\
& =\{m \geq 1 \mid \operatorname{LCM}(S) \text { divides } m \text { and } m \leq s n \text { for all } s \in S\} .
\end{aligned}
$$

Therefore, $|\bar{S}|=\left\lfloor\frac{n \cdot \min S}{\operatorname{LCM}(S)}\right\rfloor$. 
Suppose that $n=a+b \cdot \operatorname{LCM}\left(I_{k}\right)$, where $a, b \in \mathbb{N}$ with $a \leq \operatorname{LCM}\left(I_{k}\right)$. For any nonempty subset $S$ of $I_{k}$, since $\operatorname{LCM}(S)$ divides $\operatorname{LCM}\left(I_{k}\right), \frac{b \cdot \operatorname{LCM}\left(I_{k}\right) \cdot \min S}{\operatorname{LCM}(S)}$ is an integer, and we have

$$
\begin{aligned}
\left\lfloor\frac{n \cdot \min S}{\operatorname{LCM}(S)}\right\rfloor & =\left\lfloor\frac{\left(a+b \cdot \operatorname{LCM}\left(I_{k}\right)\right) \cdot \min S}{\operatorname{LCM}(S)}\right\rfloor=\left\lfloor\frac{a \cdot \min S}{\operatorname{LCM}(S)}+\frac{b \cdot \operatorname{LCM}\left(I_{k}\right) \cdot \min S}{\operatorname{LCM}(S)}\right\rfloor \\
& =\left\lfloor\frac{a \cdot \min S}{\operatorname{LCM}(S)}\right\rfloor+\frac{b \cdot \operatorname{LCM}\left(I_{k}\right) \cdot \min S}{\operatorname{LCM}(S)}=\left\lfloor\frac{a \cdot \min S}{\operatorname{LCM}(S)}\right\rfloor+b \cdot\left\lfloor\frac{\operatorname{LCM}\left(I_{k}\right) \cdot \min S}{\operatorname{LCM}(S)}\right\rfloor .
\end{aligned}
$$

This makes $d_{k}\left(a+b \cdot \operatorname{LCM}\left(I_{k}\right)\right)=d_{k}(a)+b \cdot d_{k}\left(\operatorname{LCM}\left(I_{k}\right)\right)$. If we can show that $d_{k}(n) \geq n$ for all $n$ with $n \leq \operatorname{LCM}\left(I_{k}\right)$, then we are done.

With the above preparation, we make the assumption that

$$
2 k<n \leq \operatorname{LCM}\left(I_{k}\right)
$$

for the rest of the paper, and move on.

\section{THE CASE WHEN $n>2 k$}

We start with an easy observation.

Lemma 4.1. Let $p$ and $q$ be distinct primes which are greater than $\max \{k, \sqrt{n}\}$ and less than or equal to $n$. Then for any $s, t \in \mathbb{N}$ with $s \leq\left\lfloor\frac{n}{p}\right\rfloor$ and $t \leq\left\lfloor\frac{n}{q}\right\rfloor$, we have $(s p) I_{k} \cap(t q) I_{k}=\varnothing$.

Proof. Suppose that $s p a=t q b$ for some $a, b \in I_{k}$. Then $p \mid t b$. Since $p$ is a prime larger than $k$, we must have $p \mid t$. From $t \leq\left\lfloor\frac{n}{q}\right\rfloor$, we reach a contradiction that $p q \leq t q \leq n$.

Remark 4.2. Suppose that $p$ is a prime such that $\max \{k, \sqrt{n}\}<p \leq n$. Then

$$
p I_{k} \Delta 2 p I_{k} \Delta \cdots \Delta\left\lfloor\frac{n}{p}\right\rfloor p I_{k}=p\left(I_{k} \Delta 2 I_{k} \Delta \cdots \Delta\left\lfloor\frac{n}{p}\right\rfloor I_{k}\right),
$$

which has at least $\max \left\{k,\left\lfloor\frac{n}{p}\right\rfloor\right\}$ many elements by the induction hypothesis. Combining this with Lemma 4.1 our goal is then to show that

$$
\sum_{p \in \mathcal{P}} \max \left\{k,\left\lfloor\frac{n}{p}\right\rfloor\right\} \geq n,
$$

where $\mathcal{P}$ is defined to be

$$
\mathcal{P}=\{p \mid p \text { is a prime and } \max \{k, \sqrt{n}\}<p \leq n\} .
$$

We will use the following results from number theory, where $\pi(x)$ denotes the number of primes less than or equal to $x$.

Proposition 4.3 ([2, Theorem 2 and Corollary to Theorem 3] and [5, (3.5), (3.6), (3.8)]).

(1) $2^{k} \leq \operatorname{LCM}\left(I_{k}\right) \leq 4^{k}$.

(2) $\pi(x)>x / \ln x$ for $x \geq 17$.

(3) $\pi(x)<1.25506 x / \ln x$ for $x>1$.

(4) $\pi(2 x)-\pi(x)>3 x /(5 \ln x)$ for $x>20.5$. 
4.1. The case when $\mathbf{2} \mathbf{k}<\mathbf{n} \leq \mathbf{k}^{\mathbf{2}}$. Let $\left\lceil\frac{n}{k}\right\rceil=m$. Thus, $m \geq 3$ and $k m \geq n$. Since each prime in $\mathcal{P}$ contributes at least $k$ elements to the set $D_{k \times n}$ (see Remark 4.2), we aim to show that $|\mathcal{P}| \geq m$.

Therefore, we want $\pi(n)-\pi(k) \geq m$. It is easy to verify that $d_{k}(n) \geq n$ for $k$ and $n$ with $k \leq 20$ and $2 k<n \leq k^{2}$. So we assume that $k \geq 21$.

Let $v=m-1$. Then $2 \leq v \leq k-1$ and $n \geq v k+1$, and the goal is to show that $\pi(v k+1)-\pi(k) \geq v+1$. For $v=2,3$ or 4 , we have

$$
\pi(v k+1)-\pi(k) \geq \pi(2 k)-\pi(k) \geq 3 k /(5 \ln k) \geq 3 \cdot 21 /(5 \ln 21)>4.1 .
$$

Since $\pi(v k+1)-\pi(k)$ is an integer, it is at least 5 , which is greater than $v-1$.

On the other hand, for $v \geq 5$ we have

$$
\begin{aligned}
\pi(v k+1)-\pi(k) & \geq \pi(v k)-\pi(k) \geq \frac{v k}{\ln (v k)}-\frac{1.25506 k}{\ln k} \\
& >\frac{v k}{\ln \left(k^{2}\right)}-\frac{1.3 k}{\ln k}>\frac{v k}{2 \ln k}-\frac{1.3 k}{\ln k} \\
& =\frac{k}{\ln k} \cdot\left(\frac{v}{2}-1.3\right) \geq \frac{21}{\ln 21} \cdot\left(\frac{v}{2}-1.3\right) \\
& >6 \cdot\left(\frac{v}{2}-1.3\right)>v+1 .
\end{aligned}
$$

Thus the case when $2 k<n \leq k^{2}$ is done.

4.2. The case when $\mathbf{n}>\mathbf{k}^{2}$. In this case, $\max \{k, \sqrt{n}\}=\sqrt{n}$. Let $a_{0}=n$, $a_{i}=\frac{n}{k+i}$ for $i=1, \ldots, \ell$, and $a_{\ell+1}=\sqrt{n}$, where $\ell$ is the largest integer such that $\frac{n}{k+\ell+1} \leq \sqrt{n}<\frac{n}{k+\ell}$. Here we have $\left\lfloor\frac{n}{p}\right\rfloor \leq k$ if $a_{1}<p \leq a_{0}$, and $\left\lfloor\frac{n}{p}\right\rfloor=k+i$ if $a_{i+1}<p \leq a_{i}(i=1,2, \ldots, \ell)$; therefore,

$$
\begin{aligned}
\sum_{p \in \mathcal{P}} & \max \left\{k,\left\lfloor\frac{n}{p}\right\rfloor\right\}=\sum_{\substack{\sqrt{n}<p \leq n \\
\text { is prime }}} \max \left\{k,\left\lfloor\frac{n}{p}\right\rfloor\right\} \\
& =\sum_{a_{1}<p \leq a_{0}} \max \left\{k,\left\lfloor\frac{n}{p}\right\rfloor\right\}+\sum_{a_{2}<p \leq a_{1}} \max \left\{k,\left\lfloor\frac{n}{p}\right\rfloor\right\}+\cdots+\sum_{a_{\ell+1}<p \leq a_{\ell}} \max \left\{k,\left\lfloor\frac{n}{p}\right\rfloor\right\} \\
& =\sum_{a_{1}<p \leq a_{0}} k+\sum_{a_{2}<p \leq a_{1}}\left\lfloor\frac{n}{p}\right\rfloor+\cdots+\sum_{a_{\ell+1}<p \leq a_{\ell}}\left\lfloor\frac{n}{p}\right\rfloor \\
= & \sum_{a_{1}<p \leq a_{0}} k+\sum_{a_{2}<p \leq a_{1}}(k+1)+\cdots+\sum_{a_{\ell+1}<p \leq a_{\ell}}(k+\ell) \\
= & \sum_{i=0}^{\ell}(k+i)\left(\pi\left(a_{i}\right)-\pi\left(a_{i+1}\right)\right) \\
= & \sum_{i=0}^{\ell} k\left(\pi\left(a_{i}\right)-\pi\left(a_{i+1}\right)\right)+\sum_{i=1}^{\ell} i\left(\pi\left(a_{i}\right)-\pi\left(a_{i+1}\right)\right) \\
= & \left(k \pi\left(a_{0}\right)-k \pi\left(a_{\ell+1}\right)\right)+\left(\left(\sum_{i=1}^{\ell} \pi\left(a_{i}\right)\right)-\ell \pi\left(a_{\ell+1}\right)\right) \\
= & k \pi(n)+\left(\sum_{i=1}^{\ell} \pi\left(a_{i}\right)\right)-(k+\ell) \pi(\sqrt{n}) .
\end{aligned}
$$


Using Proposition 4.3 , we have

and if $n \geq 17$,

$$
\pi(\sqrt{n})<1.25506 \cdot \frac{\sqrt{n}}{\ln \sqrt{n}}<2.52 \cdot \frac{\sqrt{n}}{\ln n},
$$

$$
\begin{aligned}
\sum_{i=1}^{\ell} \pi\left(\frac{n}{k+i}\right) & >\sum_{i=1}^{\ell} \frac{\frac{n}{k+i}}{\ln \left(\frac{n}{k+i}\right)}>\sum_{i=1}^{\ell} \frac{n}{\frac{k+i}{\ln n}}=\frac{n}{\ln n} \sum_{i=1}^{\ell} \frac{1}{k+i} \\
& >\frac{n}{\ln n}(\ln (k+\ell+1)-\ln (k+1)) .
\end{aligned}
$$

The last inequality came from $\sum_{i=1}^{\ell} \frac{1}{k+i}>\int_{k+1}^{k+\ell+1} \frac{1}{x} d x$. Also, from $\frac{n}{k+\ell+1} \leq \sqrt{n}<$ $\frac{n}{k+\ell}$ we have $k+\ell<\sqrt{n} \leq k+\ell+1$, and so $\frac{1}{2} \ln n=\ln (\sqrt{n}) \leq \ln (k+\ell+1)$. This yields

$$
\begin{aligned}
\sum_{p \in \mathcal{P}} \max \left\{k,\left\lfloor\frac{n}{p}\right\rfloor\right\} & >k \frac{n}{\ln n}+\frac{n}{\ln n}(\ln (k+\ell+1)-\ln (k+1))-(k+\ell) \frac{\sqrt{n}}{\ln n} \cdot 2.52 \\
& \geq \frac{n}{2}+\frac{n}{\ln n} \cdot(k-\ln (k+1)-2.52) .
\end{aligned}
$$

To finish the task, we need only to have $\frac{k-\ln (k+1)-2.52}{\ln n} \geq \frac{1}{2}$. To this end, we use the fact that $n \leq \operatorname{LCM}\left(I_{k}\right)<4^{k}$. So

$$
\frac{k-\ln (k+1)-2.52}{\ln n} \geq \frac{k-\ln (k+1)-2.52}{\ln \left(\operatorname{LCM}\left(I_{k}\right)\right)}>\frac{k-\ln (k+1)-2.52}{k \cdot \ln 4} .
$$

Now,

- $\frac{k-\ln (k+1)-2.52}{k \cdot \ln 4}$ is increasing for all $k$ and is more than $\frac{1}{2}$ when $k=18$.

- $\frac{k-\ln (k+1)-2.52}{\ln \left(\operatorname{LCM}\left(I_{k}\right)\right)} \geq \frac{1}{2}$ for $k=8,9, \ldots, 17$.

So, we see that, indeed,

$$
\sum_{p \in \mathcal{P}} \max \left\{k,\left\lfloor\frac{n}{p}\right\rfloor\right\} \geq n \text { when } n \geq k^{2} \geq 64 .
$$

For $k<8$, we have to check $d_{k}(n)$ for $k^{2}<n \leq \operatorname{LCM}\left(I_{k}\right)$. Since $k^{2}>\operatorname{LCM}\left(I_{k}\right)$ for $k=2,3,4$, this amounts to checking the cases $k=5$ with $25<n \leq \operatorname{LCM}(5)=$ $60, k=6$ with $36<n \leq 60$, and $k=7$ with $49<n \leq 420$. Again, a simple computer routine verifies that these are all fine. Therefore, we conclude that the Restricted 1-2-3 Conjecture is true for all $k$ and $n$ with $n \geq k^{2}$, and as well conclude our proof for the Restricted 1-2-3 Conjecture.

Finally, for the "Codes by composition" (see the introduction), one needs even more. Here, we mention as an open problem:

Extended 1-2-3 Conjecture. For every finite, nonempty subset $I$ of the natural numbers, the symmetric difference of the sets $i I_{k}, i \in I$, has at least $k$ elements.

Note that it is not true if one changes "at least $k$ elements" to "at least $k$ or $n$ elements, whichever is larger"! 


\section{REFERENCES}

[1] The GAP Group, GAP-Groups, Algorithms, and Programming, Version 4.4.12, 2008, http://www.gap-system.org.

[2] M. Nair, On Chebyshev-type inequalities for primes. Amer. Math. Monthly 89 (1982), 126129. MR643279 (83f:10043)

[3] G. Pilz, On polynomial near-ring codes, in Contributions to General Algebra, 8, HölderPichler-Tempsky, Vienna, 1992, 233-238. MR.1281844 (95e:11131)

[4] G. Robin, Estimate of the Chebyshev function $\theta$ on the $k$ th prime number and large values of the number of prime divisors function $\omega(n)$ of n. Acta Arith. 42 (1983), 367-389. MR736719 (85j:11109)

[5] J. B. Rosser and L. Schoenfeld. Approximate formulas for some functions of prime numbers. Illinois J. Math. 6 (1962), 64-94. MR0137689(25:1139)

Department of Mathematics and National Center for Theoretical Sciences (South), National Cheng Kung University, 1 University Road, Tainan 701, Taiwan

E-mail address: pyhuang@mail.ncku.edu.tw

Department of Mathematics and National Center for Theoretical Sciences (South), National Cheng Kung University, 1 University Road, Tainan 701, Taiwan

E-mail address: wfke@mail.ncku.edu.tw

Department of Algebra, Johannes Kepler Universität Linz, Altenberger Strasse 69 , 4040 Linz, Austria

E-mail address: guenter.pilz@jku.at 